OPEN ACCESS

Edited by:

Rakesh C. Kukreja,

Virginia Commonwealth University,

United States

Reviewed by:

Haobo Li,

Harvard Medical School,

United States

Mingxiang Zhang,

Shandong University, China

*Correspondence: Beibei LuO

luobeibei0608@aliyun.com

Specialty section:

This article was submitted to

Oxidant Physiology,

a section of the journal

Frontiers in Physiology

Received: 03 December 2019 Accepted: 30 March 2020

Published: 23 April 2020

Citation:

Xie Y, Huang Y, Ling X, Qin H,

Wang $M$ and Luo $B$ (2020)

Chemerin/CMKLR1 Axis Promotes

Inflammation and Pyroptosis by Activating NLRP3 Inflammasome in Diabetic Cardiomyopathy Rat.

Front. Physiol. 11:381.

doi: 10.3389/fphys.2020.00381

\section{Chemerin/CMKLR1 Axis Promotes Inflammation and Pyroptosis by Activating NLRP3 Inflammasome in Diabetic Cardiomyopathy Rat}

\author{
Yebin Xie ${ }^{1}$, Yu Huang ${ }^{1}$, Xiaoyu Ling ${ }^{1}$, Haiou Qin', Min Wang ${ }^{1}$ and Beibei Luo 1,2,3* \\ ${ }^{1}$ Department of Geriatric Cardiology, The First Affiliated Hospital of Guangxi Medical University, Nanning, China, ${ }^{2}$ Guangxi \\ Key Laboratory of Precision Medicine in Cardio-Cerebrovascular Diseases Control and Prevention, The First Affiliated \\ Hospital of Guangxi Medical University, Nanning, China, ${ }^{3}$ Guangxi Clinical Research Center for Cardio-Cerebrovascular \\ Diseases, The First Affiliated Hospital of Guangxi Medical University, Nanning, China
}

Chemerin and its receptor CMKLR1 (a G-protein-coupled receptor) are inducers of inflammation, and play an important role in diabetic cardiomyopathy (DCM). In this study, we investigated the role of the chemerin/CMKLR1 axis in mediating inflammation and cell death in DCM. Sprague-Dawley rats, treated with a high-fat diet and low-dose of streptozotocin, were used as a DCM model. CMKLR1 expression was knocked down by siRNA (CMKLR1-siRNA) to evaluate the role of CMKLR1 in DCM. Chemerintreated $\mathrm{H} 9 \mathrm{c} 2$ cells were used to investigate the factors acting downstream of the chemerin/CMKLR1 axis. LDH release and EthD-III staining were used to measure the ratio of cell death in vitro. CMKLR1-siRNA and siRNA against nucleotide-binding oligomerization domain-like receptors 3 (NLRP3-siRNA) were used to explore the mechanism underlying chemerin-induced inflammation and cell death. The results showed that the expression of chemerin, CMKLR1, NLRP3, pro-caspase-1, activated caspase-1, and mature IL-1 $\beta$ was increased in the DCM model rat. Myocardium of DCM model rats exhibited fibrosis, hypertrophy, a disorganized ultrastructure, and impaired function. Pyroptosis was observed in vivo and in vitro. Silencing of CMKLR1 in vivo attenuated the expression of NLRP3 and activated caspase- 1 and IL-1 $\beta$. CMKLR1-siRNA treatment attenuated cardiac inflammation, fibrosis, hypertrophy, and pyroptosis, and improved cardiac function in vivo. Silencing of either CMKLR1 or NLRP3 suppressed the levels of activated caspase-1, IL-1 $\beta$, and pyroptosis; however, silencing of both CMKLR1 and NLRP3 further decreased the levels of mature IL$1 \beta$ and pyroptosis. Overall, the results showed that the chemerin/CMKLR1 axis contributed to the development of DCM and that the NLRP3 inflammasome mediated the chemerin/CMLR1-induced inflammation and pyroptosis. These data indicate that silencing of the CMKLR1 gene might exert a protective effect against DCM.

Keywords: chemerin, CMKLR1, NLRP3 inflammasome, pyroptosis, inflammation 


\section{INTRODUCTION}

The number of patients with diabetes worldwide is projected to reach approximately 642 million by 2040 (Ogurtsova et al., 2017). Cardiovascular disease is the most common complication of diabetes, and often leads to heart failure (Yap et al., 2019). Diabetic cardiomyopathy (DCM), characterized by disordered myocardial structure and function, is an important cause of heart failure among diabetic patients (Marwick et al., 2018). Previous research has indicated that inflammation is a critical inducer of cardiac remodeling, including cell death, fibrosis, and hypertrophy, which results in heart failure (Wang et al., 2016; Jin et al., 2017; Deckx et al., 2018; Wu et al., 2019; Yang L. et al., 2019). However, the mechanisms underlying the inflammatory processes in DCM remain unknown.

Chemerin, also known as tazarotene-induced gene 2 protein or retinoic acid receptor responder protein 2 , is secreted by various cell types, including adipocytes, epithelial and endothelial cells, and fibroblasts (Helfer and Wu, 2018). Chemerin is secreted as an inactive precursor, pro-chemerin, which can be converted into the active form of chemerin following protease cleavage of the C-terminal domain. The main function of chemerin was initially described as the regulator of glucolipid metabolic processes and inflammatory responses (Helfer and $\mathrm{Wu}, 2018$ ). Recently, however, chemerin was reported to be a key regulator of cardiovascular disease, including hypertension, coronary atherosclerosis disease, and myocardial infarction (Kadoglou et al., 2015). In patients with type 2 diabetes, elevated serum levels of chemerin have been associated with heart failure (Zhou et al., 2019) while chemerin was shown to induce murine cardiomyocyte apoptosis through inflammatory processes (Rodriguez-Penas et al., 2015). These results suggest that chemerin plays an important role in DCM by inducing cardiac inflammation.

Membrane-localized receptors for chemerin include G-protein-coupled chemokine-like receptor 1 (CMKLR1), G protein-coupled receptor 1 (GPR1), and chemokine (CC motif) receptor-like 2 (CCRL2) (Kaur et al., 2018). However, only CMKLR1 appears to be critical for chemerin-induced inflammation (Mariani and Roncucci, 2015). Chemerin binding to GPR1 produces only a weak effect in modulating calcium mobilization, while CCRL2 does not activate downstream signaling cascades (Stojek, 2017). In contrast, CMKLR1 has been reported to mediate chemerin-promoted inflammation in diabetic complication (Neves et al., 2018a,b; Zou et al., 2019). However, the role of the chemerin/CMKLR1 axis in DCM, as well as the associated underlying molecular mechanisms, remain unclear.

The NACHT, LRR, and PYD domains-containing protein 3 (NLRP3) inflammasome has been reported to regulate inflammatory processes in DCM. The NLRP3 inflammasome consists of NLRP3, apoptosis-associated speck-like protein containing a CARD (ASC), and the serine protease caspase-1 (Zhang et al., 2018; Kelley et al., 2019; Yang F. et al., 2019). Once activated, NLRP3 interacts with ASC, which induces the cleavage of pro-caspase- 1 and formation of an active caspase- 1 tetramer composed of two p20 and two p10 subunits (Kelley et al., 2019).
The caspase- 1 tetramer then cleaves pro-IL-1 $\beta$, converting it into IL-1 $\beta$, a critical step in myocardial apoptosis, hypertrophy, and fibrosis (Wang et al., 2016; Jin et al., 2017; Deckx et al., 2018; Zhang et al., 2018; Yang L. et al., 2019).

Activated caspase-1 can induce an inflammatory form of programmed cell death termed "pyroptosis" (Lacey et al., 2018), which shows characteristics typical of apoptosis and necrosis. Similar to apoptosis, cells undergoing pyroptosis incur DNA damage and become TUNEL-positive; and as with necrosis, pyroptosis exhibits membrane pore formation, release of proinflammatory cytoplasmic content, and cell rupture (Liu et al., 2016). EthD-III, a type of cell membrane-impermeant nucleic acid dye, stains pyroptotic cells by entering through the membrane pores, but does not stain cells with intact membranes (Pan et al., 2018; Wang X. et al., 2019). The permeability of pyroptotic cell membranes can also be determined through the release of the cytoplasmic enzyme lactose dehydrogenase (LDH) (Ding et al., 2019; Gu et al., 2019). Recent studies showed that pyroptosis also occurs in cardiomyocytes (Pan et al., 2018; Meng et al., 2019; Wang X. et al., 2019). In our previous study, we showed that NLRP3 inflammasomeinduced caspase-1 activation promotes cardiomyocyte pyroptosis in DCM. However, the regulatory mechanism underlying NLRP3 inflammasome activation in DCM remains unknown.

The expression of chemerin and NLRP3 was shown to be upregulated in lung tissue following Limb I/R, possibly due to early responses to inflammation (Zou et al., 2019). Additionally, the chemerin/CMKLR1 axis was shown to induce the formation of the NLRP3 inflammasome as well as the expression of several proinflammatory cytokines in Kupffer cells (Zhang et al., 2017). However, little is known about the role of the chemerin/CMKLR1 axis in the regulation of the NLRP3 inflammasome in DCM.

The aim of the present study was to determine the role of the chemerin/CMKLR1 axis in DCM rats, and whether the NLRP3 inflammasome is an important mediator of the chemerin/CMKLR1 signaling-induced inflammatory effects in DCM.

\section{MATERIALS AND METHODS}

\section{Animals}

Forty Sprague-Dawley rats (100-120 g) were divided into four groups ( $n=10$ per group) as follows: a control group (Ctrl), diabetic cardiomyopathy (DCM) group, DCM + negative control lentivirus (DCM+NC) group, and DCM+CMKLR1-siRNA group. All the rats were maintained at $22^{\circ} \mathrm{C}$ with $12 \mathrm{~h}$ light-dark cycles. The control group was fed a basal diet while the other groups were fed a high-fat diet ( $16 \%$ fat and $0.25 \%$ cholesterol). After 4 weeks, all groups underwent an intraperitoneal glucose tolerance test (IPGTT) and intraperitoneal insulin tolerance test (IPITT) to identify insulin resistance. To induce diabetes, rats in the DCM, DCM+NC, and DCM+CMKLR1-siRNA groups were administered a single intraperitoneal streptozotocin injection (STZ, $35 \mathrm{mg} / \mathrm{kg}$; Solarbio, Beijing, China). Blood glucose levels were analyzed by blood glucose meter 1 week after STZ treatment (Roche, Switzerland). Rats with fasting 
blood glucose levels $\geq 11.1 \mathrm{mmol} / \mathrm{L}$ were considered diabetic. CMKLR1-siRNA treatment was carried out 8 weeks after STZ injection as this is when cardiac dysfunction occurs in diabetic rats (Lacombe et al., 2007; Ti et al., 2011). Rats from the DCM+NC and DCM+CMKLR1-siRNA groups were administered NC lentivector or CMKLR1-siRNA at a total dose of $1 \times 10^{8} \mathrm{TU} /$ rat (Genechem, China) by jugular vein injection. The sequences of the siRNAs used in this study were CMKLR1-siRNA: 5'-CAGUGAACAUGGUCUGGUU[dT][dT]3' (antisense); and NC: 5'-GCGCCAGUGGUACUUAAUATT-3' (antisense). All the rats were euthanized under deep anesthesia 8 weeks after lentivector injection. Hearts were excised and immediately frozen to determine the transfection efficiency using fluorescence microscopy. The study protocol was approved by the Institutional Animal Care and Use Committee of the Guangxi Medical University. The study was performed in accordance with the National Institutes of Health (NIH) Guidelines for the Care and Use of Laboratory Animals.

\section{Echocardiography}

Sixteen weeks after STZ injection, the rats were anesthetized with $10 \%$ chloral hydrate and transthoracic echocardiography was performed using the Vevo 770 imaging system with a RMB710 transducer (VisualSonics, Canada). The echocardiography parameters analyzed included left ventricular end-diastolic dimension (LVEDd), left ventricular ejection fraction (LVEF), fractional shortening (FS), and peak $\mathrm{E}$ to peak A ratio (E/A).

\section{Blood Pressure Assessment}

Sixteen weeks after STZ injection, systolic blood pressure (SBP) and diastolic blood pressure (DBP) were evaluated in conscious rats using a tail-cuff system (Kent Scientific, United States).

\section{Histological Examination}

The hearts were arrested with $10 \% \mathrm{KCl}$, and images of whole hearts were captured using a Canon camera (Tokyo, Japan). Sections $(4 \mu \mathrm{m})$ were stained with hematoxylin and eosin (H\&E). Cross section measurements were performed at the level of the nucleus in longitudinally sectioned myocytes. Images were obtained under a microscope (Olympus, Japan). Data were analyzed by Image-Pro Plus 6.0 (Media Cybemetics, United States).

\section{Immunohistochemistry}

For immunohistochemistry, $4-\mu \mathrm{m}$ tissue sections were incubated with primary antibodies against chemerin (1:100; Abcam, United Kingdom) and CMKLR1 (1:200; Abcam, United Kingdom) at $4^{\circ} \mathrm{C}$ overnight. Then, the sections were washed with phosphate-buffered saline (PBS) and incubated in secondary antibody for $30 \mathrm{~min}$ at $37^{\circ} \mathrm{C}$, developed with daminobenzidine (DAB), and counterstained with hematoxylin. The images were obtained under a microscope (Olympus, Japan). Data were analyzed by Image-Pro Plus 6.0 (Media Cybemetics, United States).

\section{Masson's Trichrome Staining}

Masson's trichrome staining was performed to determine the levels of collagen deposition in the hearts. Paraffin sections were stained using a Masson's Trichorme Stain Kit (Solarbio, China). Images were obtained under a microscope (Olympus, Japan). Fibrotic areas were analyzed by Image-Pro Plus (Media Cybemetics, United States).

\section{TUNEL Assay}

Paraffin sections were treated with xylene, rehydrated through a graded alcohol series, and then placed in 3\% hydrogen peroxide in methanol for $10 \mathrm{~min}$ at room temperature. After adding the equilibration buffer, sections were treated with TdT-enzyme for $1 \mathrm{~h}$ at $37^{\circ} \mathrm{C}$. The samples were then incubated with digoxigeninconjugated antibodies for $30 \mathrm{~min}$ at $37^{\circ} \mathrm{C}$. DAB was used as the staining agent. All procedures were performed following the manufacturer's instructions (Millipore, United States).

For the TUNEL assay, cells were cultured on chamber slides, fixed in $4 \%$ paraformaldehyde for $30 \mathrm{~min}$, and then permeabilized with Immunol Staining Wash Buffer (Beyotime, China) for 2 min on ice. The subsequent steps were as described in the section of TUNEL assay of paraffin samples. Images were captured under a microscope (Olympus, Japan). The TUNEL-positive ratio was assessed by Image-Pro Plus (Media Cybemetics, United States).

\section{Transmission Electron Microscopy}

Approximately $1 \mathrm{~mm}^{3}$ of myocardium was obtained from the left ventricle and fixed in $2.5 \%$ glutaraldehyde in $0.1 \mathrm{~mol} / \mathrm{L}$ sodium cacodylate buffer ( $\mathrm{pH}$ 7.4) for $120 \mathrm{~min}$, postfixed in buffered osmic acid, dehydrated in different alcohol concentrations, and embedded in Epon 812 mixture. Tissue sections $(2 \mu \mathrm{m})$ were rinsed overnight in $0.1 \mathrm{~mol} / \mathrm{L}$ phosphate buffer, postfixed for $2 \mathrm{~h}$ in $1 \%$ osmium tetroxide, dehydrated, and then embedded in an Araldite mixture. The sections were stained with uranyl acetate and lead citrate. Finally, images were obtained by transmission electron microscopy (TEM) (H-7650, HITACHI, Japan).

\section{Quantitative Real-Time RT-PCR}

Total RNA was extracted from the left ventricle using TRIzol reagent (Invitrogen, CA, United States). Extracted RNA was reverse transcribed using a RevertAid First Strand cDNA Synthesis Kit (Thermo Fisher Scientific, United States). qPCR was performed using the Fast SYBR Green Master Mix (Thermo Fisher Scientific). The following primers were used: Chemerin forward, 5'-AAGGACTGGAAAAAGCCAGAG$3^{\prime}$ and reverse, 5'-TCCGGCCTAGAACTTTACCC-3'; CMKLR1 forward, 5'-GACCGGATTAGAACCCCAGT-3' and reverse, $5^{\prime}$-AAAACCCCAAACCCATTAGC- $3^{\prime}$; and $\beta$-actin forward, 5'-AGACCTTCAACACCCCAG-3' and reverse, 5' CACGATTTCCCTCTCAGC-3'. Relative mRNA expression was calculated by the $2^{-\triangle \triangle \mathrm{CT}}$ method.

\section{Western Blot}

Total protein was extracted from left ventricular or cell lysates using RIPA buffer and the content was measured using the Bicinchoninic acid assay (Beyotime). Protein samples were 
separated by 10-12\% SDS-PAGE, transferred to nitrocellulose membranes (Millipore), and incubated with primary antibody. The following primary antibodies were used: anti-chemerin (1:700), anti-CMKLR1 (1:500), NLRP3 (1:500), caspase-1 (1:500), IL-1 $\beta(1: 1,000)$ (all from Abcam), and $\beta$-actin (1:1,000, Santa Cruz Biotechnology, United States). Bands were revealed using a chemiluminescence reagent kit (Millipore) and quantified by densitometric analysis (Quantity One, Bio-Rad, United States).

\section{Enzyme-Linked Immunosorbent Assay}

Fasting blood samples were collected from all groups after overnight fasting. Serum was obtained by centrifugation at $500 \times g$ for $10 \mathrm{~min}$ and then stored at $-80^{\circ} \mathrm{C}$ until analysis. The rat insulin ELISA kit was purchased from Jiancheng, Nanjing (China). The murine chemerin ELISA kit was purchased from R\&D Systems (United Kingdom). The serum levels of insulin and chemerin were determined according to the manufacturer's instructions.

\section{Cell Culture and Treatment}

H9c2 cells (American Type Culture Collection) were cultured in normal glucose $(5.5 \mathrm{mM})$ Dulbecco's modified Eagle's medium (DMEM) supplemented with $10 \%$ fetal bovine serum (FBS) at $37^{\circ} \mathrm{C}$ and $5 \% \mathrm{CO}_{2}$. During the treatment period, and before stimulation, $\mathrm{H} 9 \mathrm{c} 2$ cells were synchronized by serum starvation for $12 \mathrm{~h}$. Chemerin was added to the medium at a concentration of $0-300 \mathrm{ng} / \mathrm{mL}$ for $0-48 \mathrm{~h}$ for evaluation of lactate dehydrogenase $(\mathrm{LDH})$ release.

\section{Lentivirus Transfection}

NC lentivirus, lentivirus-CMKLR1-siRNA, or lentivirusCMKLR1-siRNA + lentivirus-NLRP3-siRNA were transfected into $\mathrm{H} 9 \mathrm{c} 2$ cells at a multiplicity of infection (MOI) of 10. The sequence of the siRNA for rat NLRP3 was $5^{\prime}$ CCUGUCUUUGCCGTAGAUUACCGUAAG-3' (antisense). Transfection efficiency was assessed by fluorescence microscopy. Cells were used only if CMKLR1 and/or NLRP3 mRNA levels were decreased by at least $70 \%$ compared with the control. Three parallel experiments were performed.

\section{LDH Release and EthD-III Staining}

Supernatants from the treated cells were collected and centrifuged to remove solid particles. Lactate, INT, and diaphorase were mixed to produce the reaction mixture (Beyotime). Supernatants from each group were transferred into new 96-well plates and mixed with the reaction mixture for $30 \mathrm{~min}$ at room temperature. Serum-free medium was used as the $0 \%$ control and lysate of untreated cells was used as the $100 \%$ positive control. The absorbance value was analyzed at $490 \mathrm{~nm}$. LDH release from each group was determined based on a standard curve.

EthD-III (Viability/Cytotoxicity Assay Kit, Biotium, United States) can enter into dead cells through membrane pores, producing an intense red fluorescence. Briefly, cells were incubated with $2 \mu \mathrm{M}$ EthD-III at $37^{\circ} \mathrm{C}$ for $45 \mathrm{~min}$ and then stained with DAPI. Cells were visualized at 522/593 nm by confocal microscopy (LSM710, Carl Zeiss, Germany).

\section{Statistical Analysis}

SPSS v18.0 (SPSS, Chicago, IL, United States) was used for data analysis. Data are shown as means \pm SEM. Differences among experimental groups were analyzed by ANOVA, followed by the Tukey-Kramer post hoc test and independent samples $t$-test. $p<0.05$ was considered significant.

\section{RESULTS}

\section{Diabetes Increased Chemerin and CMKLR1 Expression, and Knockdown of CMKLR1 by CMKLR1-siRNA in DCM}

\section{Rats}

When compared with control rats, DCM rats showed increased serum levels of chemerin (Figure 1A; $p<0.01$ ). The mRNA and protein levels of chemerin and CMKLR1 in left ventricle was increased in DCM rats than those in control (Figures 1C-G; $p<0.05 \sim p<0.01$ ). Four weeks after CMKLR1-siRNA treatment, transfection efficiency was evaluated in DCM rats received normal saline injection and DCM rat received CMKLR1-siRNA-injection. In the CMKLR1siRNA group, more than $50 \%$ of myocytes were successfully transfected (Figure 1B). Western blot analysis revealed that CMKLR1-siRNA inhibited CMKLR1 upregulation in DCM rats (Figures 1E,G; $p<0.01$ ), whereas it had no effect on chemerin (Figures 1E,F). Similar results were obtained with immunohistochemical staining (Figure 1H). In addition, immunohistochemistry clearly indicated the cytosolic expression of chemerin and membrane-localized expression of CMKLR1 in DCM rats when compared with control rats (Figure $\mathbf{1 H}$ ).

\section{CMKLR1 Gene Silencing Inhibited NLRP3 Inflammasome Activation in DCM Rats}

We then analyzed the protein expression of NLRP3, procaspase-1, activated caspase-1, and activated IL-1 $\beta$ in the rat myocardium, and found that the expression levels of all these proteins were higher in DCM rats than in control rats (Figure 2; $p<0.05 \sim p<0.01)$. CMKLR1-siRNA suppressed the DCMinduced increase in the protein levels of NLRP3, activated caspase-1, and mature IL-1 $\beta$, but not that of pro-caspase- 1 (Figure $2 ; p<0.05 \sim p<0.01$ ).

\section{CMKLR1 Gene Silencing Reversed the DCM-Induced Myocardial Remodeling}

Cardiac hypertrophy was observed in DCM rats (Figures 3A,B). The cross-section area of left ventricle cardiomyocyte from DCM rats was increased compared with the control group (Figure 3F; $p<0.01)$. TUNEL staining results showed that the ratio of dead cells in DCM group was higher than that of the control group (Figures 3D,H; $p<0.01$ ). The level of interstitial cardiac fibrosis was markedly higher in the DCM group than in the controls (Figures 3C,G; $p<0.01$ ). Symmetric myofibrils, well-organized $\mathrm{Z}$ lines with sarcomeres, and packed mitochondria beside the fibers could be observed in the cardiomyocytes of control rats 


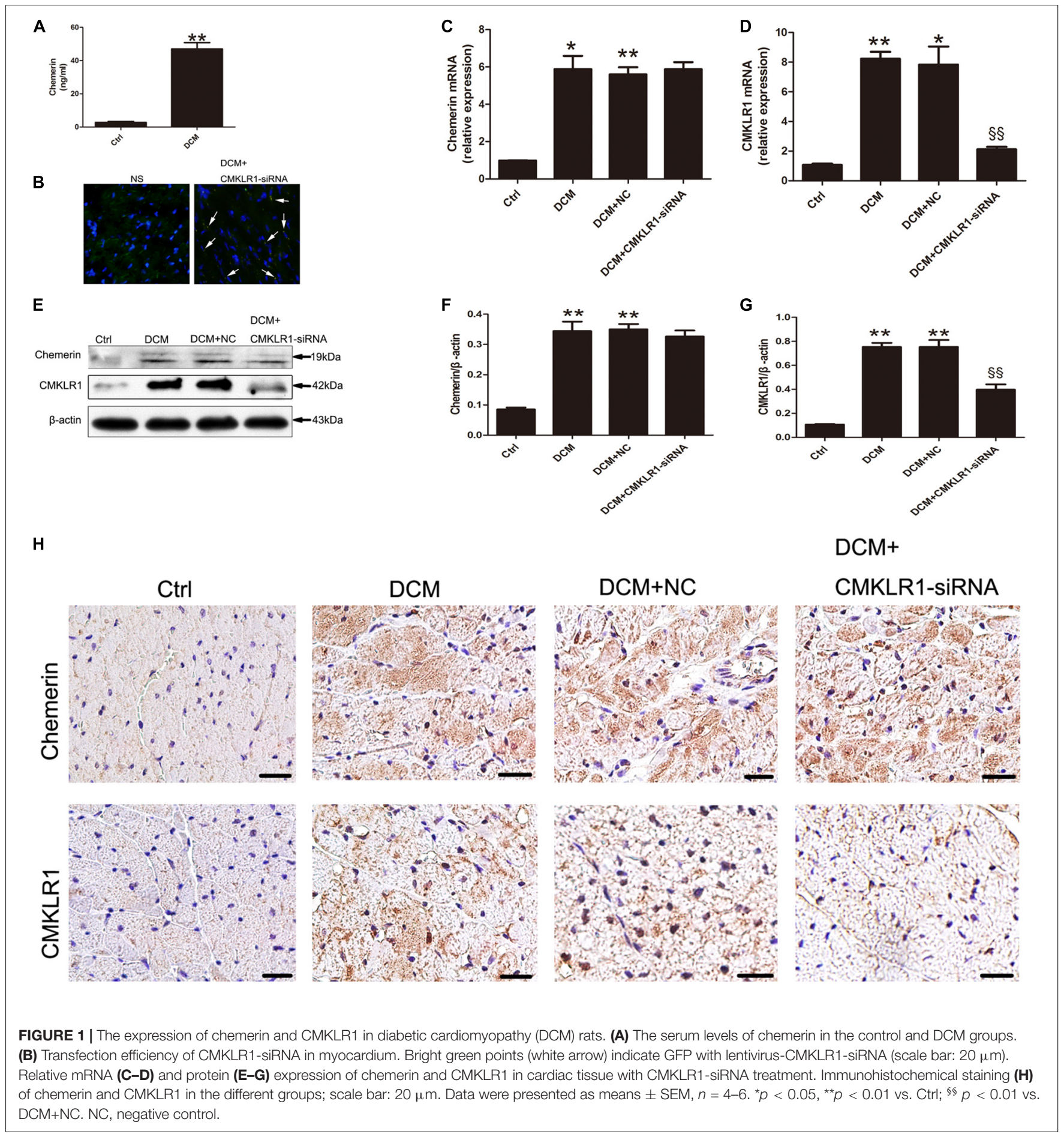

(Figure 3E). In contrast, DCM rats exhibited destruction of myofibrils, swollen mitochondria with disorganized cristae, and lipid accumulated (Figure 3E).

With CMKLR1 gene silencing, the myocardiocyte crosssection area, cardiac fibrosis area, and TUNEL-positive ratio were reduced in the DCM+CMKLR1-siRNA group compared with the NC group (Figures 3B-D,F-H; $p<0.05 \sim p<0.01$ ). CMKLR1-siRNA treatment reversed the changes in myofibrils, mitochondria, and lipid accumulation in the diabetic rats (Figure 3E).

\section{CMKLR1 Gene Silencing Improved Cardiac Dysfunction in DCM Rats}

Echocardiography showed that LVEDd was larger in DCM rats than in controls (Figures 4A,B; $p<0.05$ ). EF, 


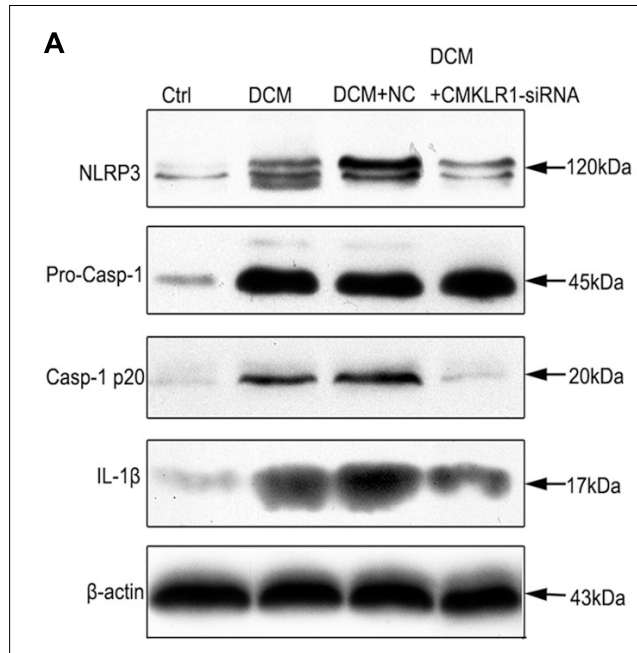

D

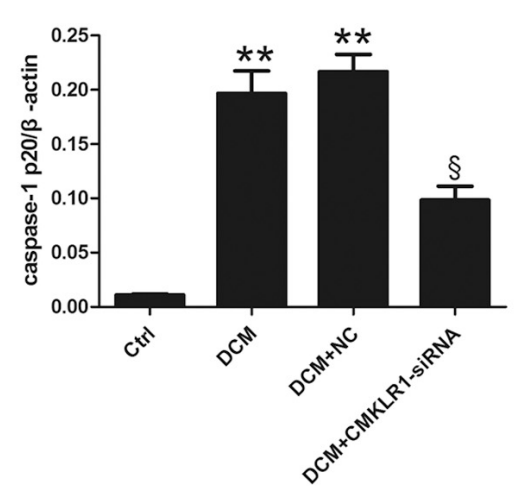

B

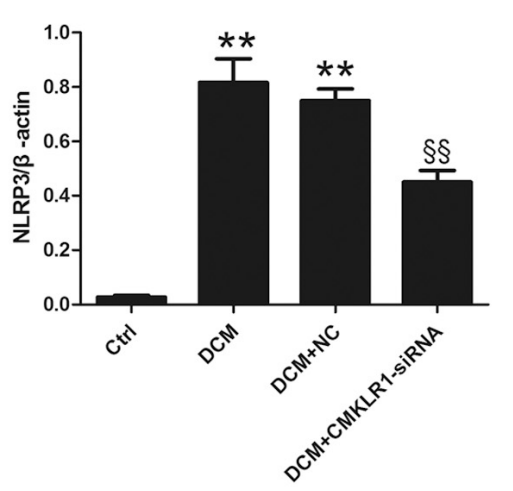

C

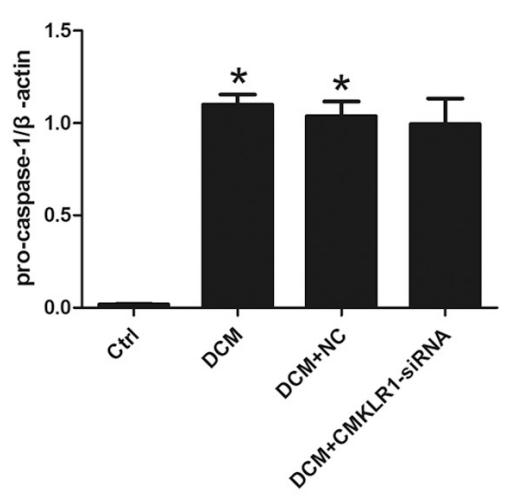

E

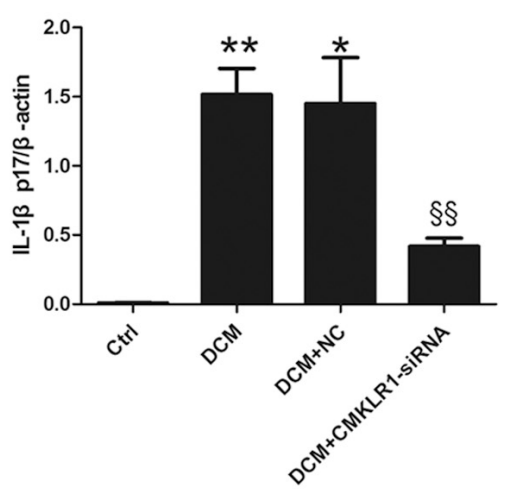

FIGURE 2 | CMKLR1 gene silencing inhibited the activation of the NLRP3 inflammasome in a rat model of diabetic cardiomyopathy (DCM). (A-E) Relative protein levels of NLRP3, pro-caspase-1, activated caspase-1, and mature IL-1 $\beta$ in the left ventricle of rats in the different groups. Data were presented as means \pm SEM from three independent experiments. ${ }^{*} p<0.05,{ }^{* *} p<0.01$ vs. Ctrl; ${ }^{\S} p<0.05,{ }^{\circledR} p<0.01$ vs. DCM+NC. NC, negative control.

$\mathrm{FS}$, and $\mathrm{E} / \mathrm{A}$ were lower in DCM rats than in controls (Figures 4A,C-E; $p<0.05 \sim p<0.01$ ). Blood pressure analysis showed that SBP and DBP were both lower in DCM rats than in controls (Figure 4F; $p<0.01$ ). Compared with the DCM+NC group, DCM+CMKLR1siRNA group exhibited increased EF, FS, and E/A, and decreased LVEDd (Figures 4A-E; $p<0.05 \sim p<0.01$ ); however, no differences were observed in SBP or DBP between the two groups (Figure 4F).

\section{Chemerin Induced LDH Release in H9c2 Cardiomyocytes}

$\mathrm{H} 9 \mathrm{c} 2$ cardiomyocytes were incubated with different concentrations of chemerin $(0,1,10,100$, or $300 \mathrm{ng} / \mathrm{mL})$ for $24 \mathrm{~h}$. LDH release was higher in the $10 \mathrm{ng} / \mathrm{mL}$-treated group than in the control (Figure 5A; $p<0.05$ ) and peaked with $100 \mathrm{ng} / \mathrm{mL}$ chemerin treatment (Figure 5A; $p<0.05$ ). The increase in the levels of $\mathrm{LDH}$ released from $\mathrm{H} 9 \mathrm{c} 2$ cardiomyoblasts treated with $100 \mathrm{ng} / \mathrm{mL}$ became significant after $12 \mathrm{~h}$, and peaked at $24 \mathrm{~h}$ (Figure 5B; both $p<0.05$ ). Based on these results,
H9c2 cells were treated with $100 \mathrm{mg} / \mathrm{mL}$ chemerin for $24 \mathrm{~h}$ for subsequent experiments.

\section{Chemerin-Induced NLRP3 Inflammasome Activation Was Mediated by CMKLR1 and NLRP3}

Chemerin treatment led to increased expression of mature caspase- 1 and IL-1 $\beta$, both of which are markers of NLRP3 inflammasome activation. Compared with the NC, CMKLR1siRNA treatment decreased the protein levels of CMKLR1 in chemerin-treated H9C2 cells (Figures 6A,B; $p<0.01$ ). In addition, CMKLR1-siRNA attenuated the chemerin-induced expression of NLRP3, caspase-1, and IL-1 $\beta$ (Figures 6A,CE; $p<0.05 \sim p<0.01)$. NLRP3-siRNA treatment suppressed the chemerin-induced protein levels of activated caspase-1 and IL-1 $\beta$ when compared with the NC (Figures 6A,C-E; $p<0.05 \sim p<0.01)$. However, NLRP3-siRNA treatment had no effect on the protein level of CMKLR1 (Figures 6A,B). Treatment with siRNAs targeting both CMKLR1 and NLRP3 further decreased the protein levels of activated IL-1 $\beta$ compared 


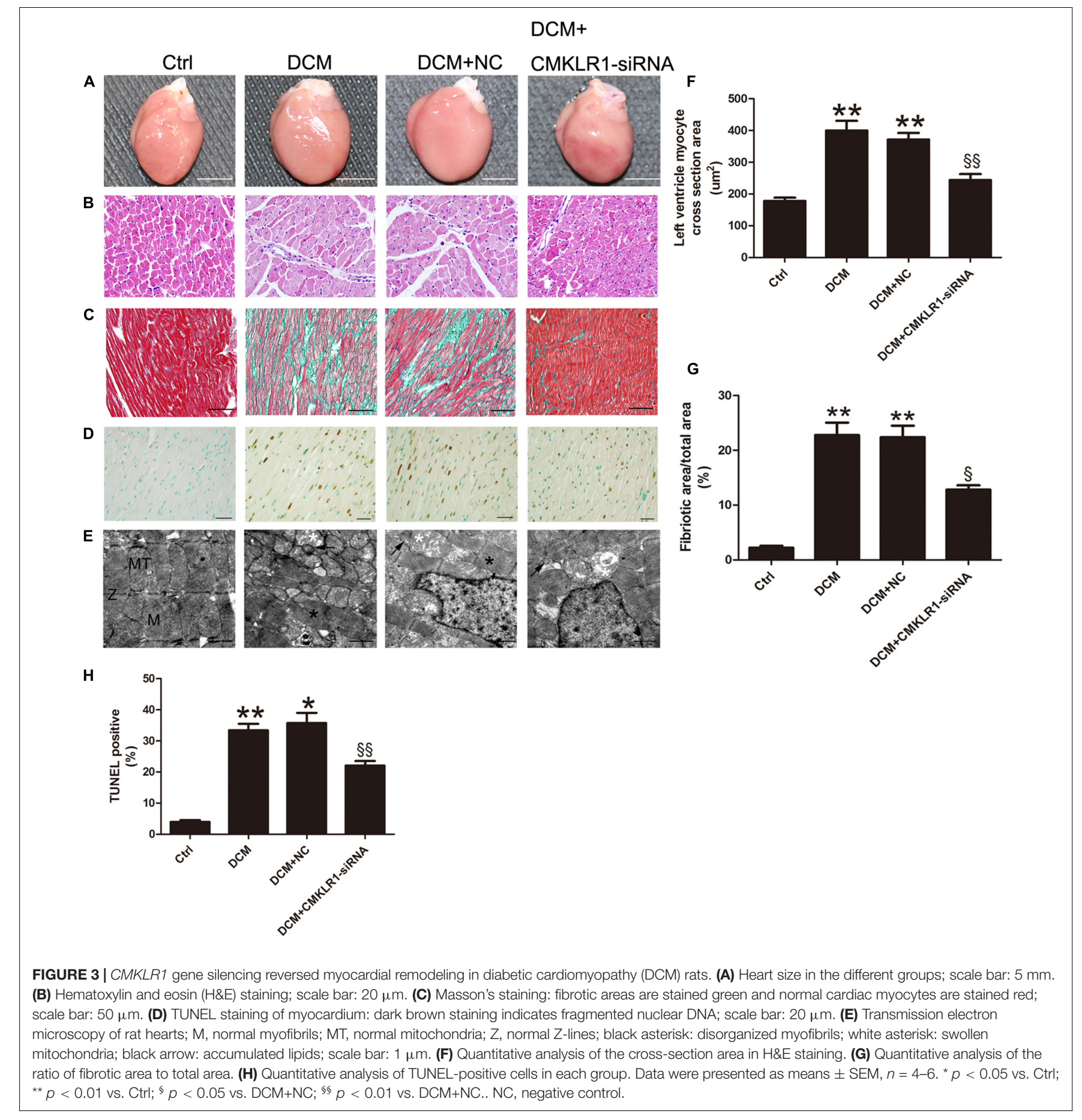

with treatment with either siRNA alone (Figures 6A,C-E; $p<0.05 \sim p<0.01$ ).

\section{NLRP3 Was Involved in Chemerin/CMKLR1-Induced Pyroptosis}

Results of the EthD-III staining and the LDH release assay showed that cell death and the number of cells with damaged membranes were increased in the chemerin-treated group compared with the control group (Figures 7A,B,D; both $p<0.01)$. Moreover, TUNEL staining results indicated that the levels of cell death associated with nuclear DNA damage were higher in the chemerin-treated group than in the control group (Figures 7A,C; $p<0.01$ ). To explore the function of NLRP3 in chemerin/CMKLR1-induced H9c2 cell death, we knocked down the expression of CMKLR1, NLRP3, and CMKLR1+NLRP3 by transfection with the respective siRNAs. Following CMKLR1 knockdown, the ratio of dead cells, as detected by EthD-III, 
A
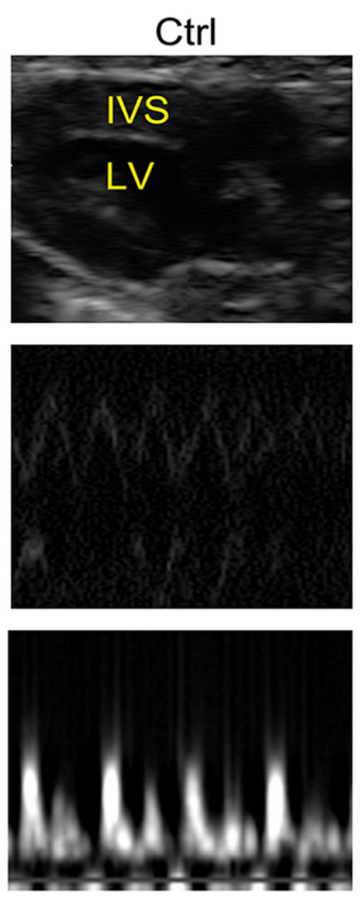

B

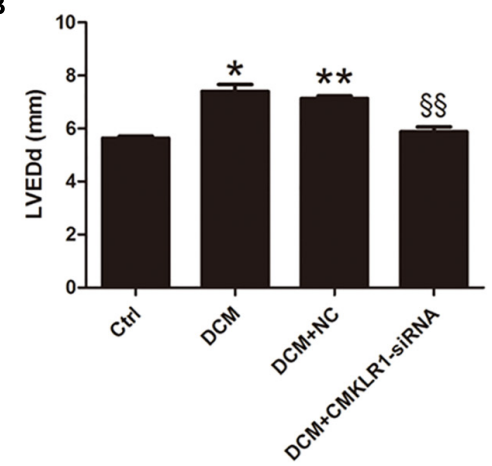

E

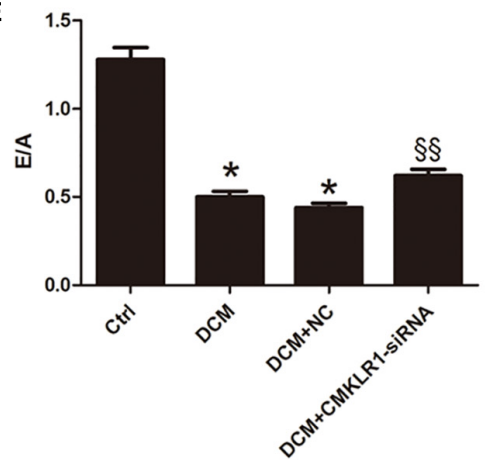

$\mathrm{DCM}+$
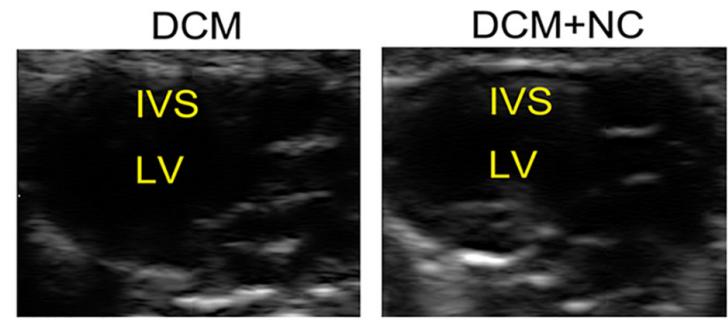

CMKLR1-SiRNA
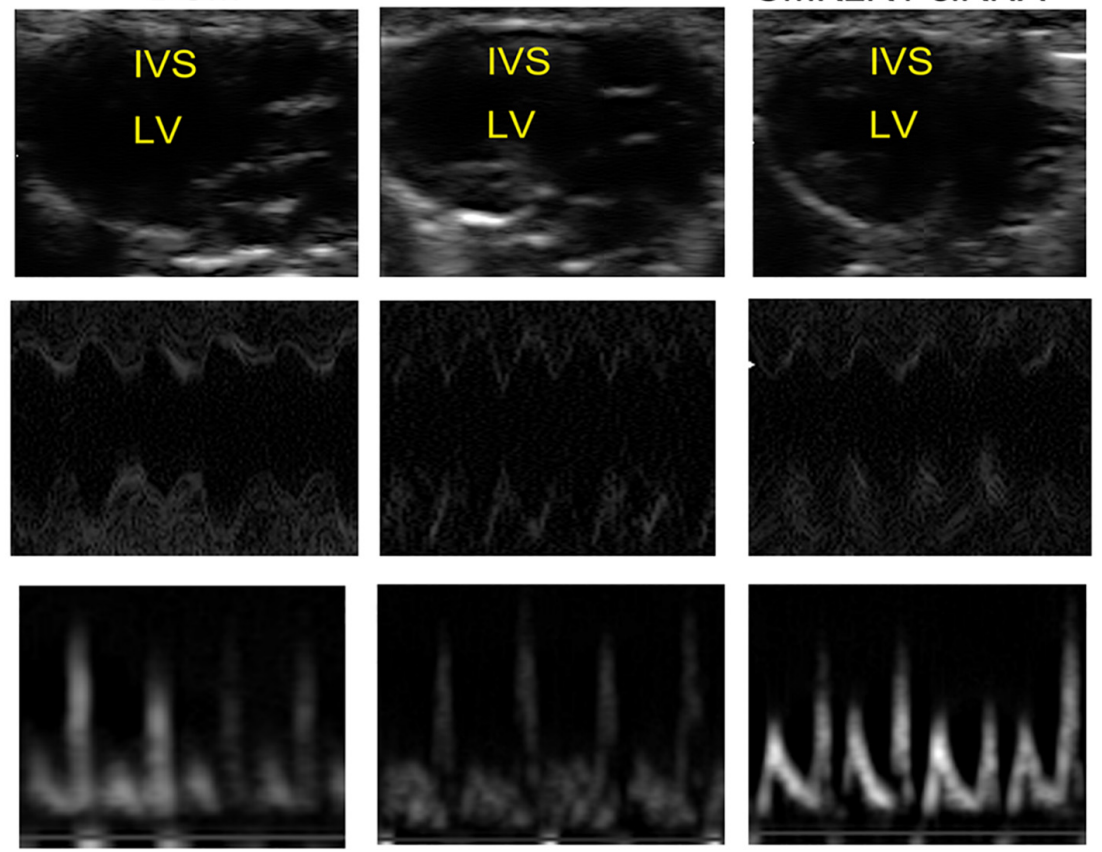

C
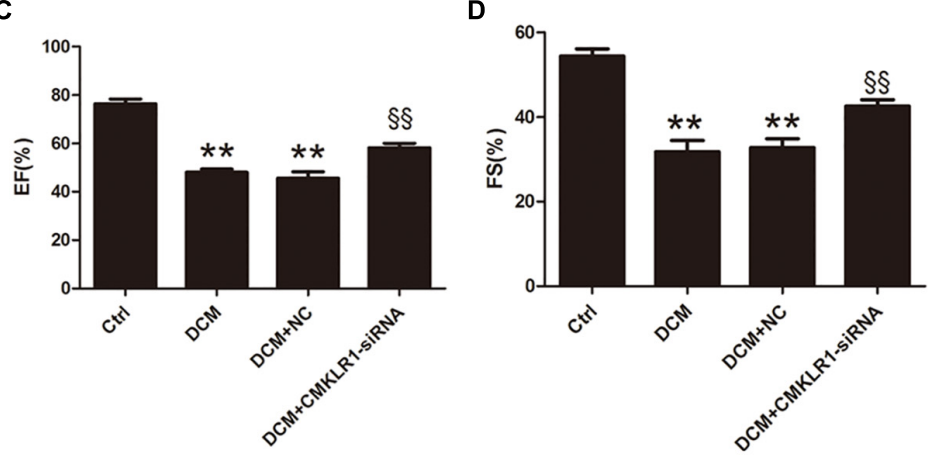

$\mathbf{F}$

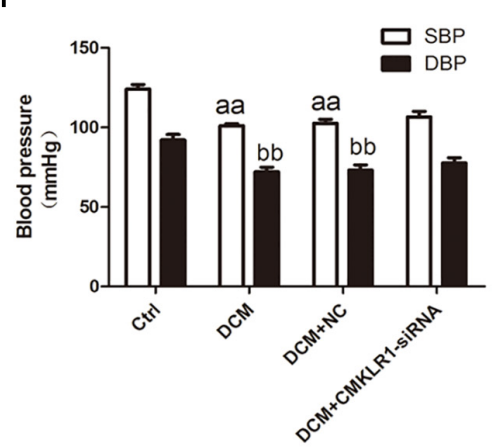

FIGURE 4 | CMKLR1 gene silencing improved cardiac dysfunction in diabetic cardiomyopathy (DCM) rats. (A) The cardiac function of rats in the different groups shown by echocardiography. Representative images of 2D echocardiograms, M-mode echocardiograms, and pulse-wave Doppler echocardiograms of mitral inflow. (B-E) Evaluation of LVEDd, EF, FS, and E/A. (F) SBP and DBP of each group at the end of study. Data were presented as means \pm SEM, $n=6$. ${ }^{*} p<0.05$, ${ }^{* \star} p<0.01$ vs. Ctrl; ${ }^{\circledR S} p<0.01$ vs. DCM+NC, ${ }^{a a} p<0.01$ vs. SBP of Ctrl, ${ }^{b b} p<0.01$ vs. DBP of Ctrl; NC, negative control; LV, left ventricle; IVS, interventricular septum; LVEDd, left ventricular end-diastolic dimension; EF, left ventricular ejection fraction; FS, fractional shortening; E/A, peak E to peak A ratio; SBP, systolic blood pressure; DBP, diastolic blood pressure. 
A

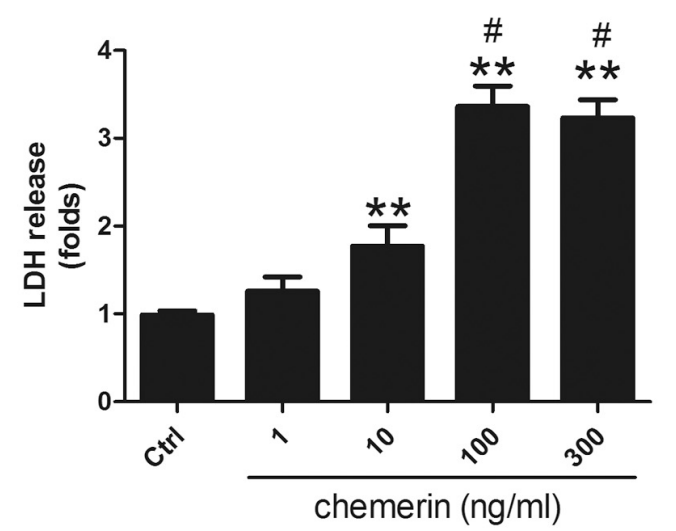

B

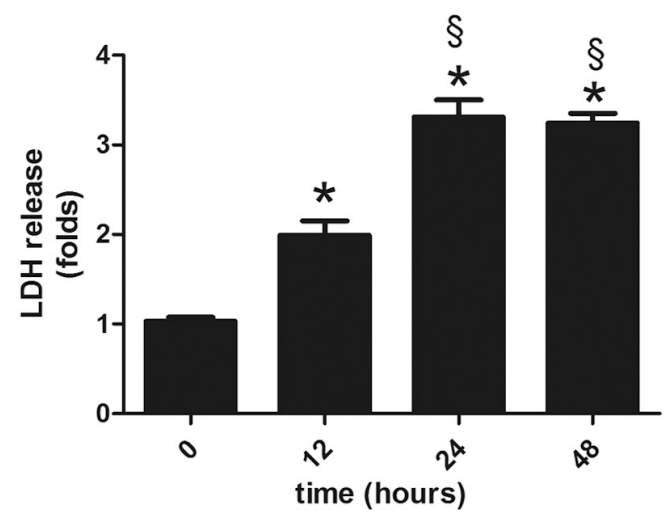

FIGURE 5 | Chemerin induced LDH release in H9c2 cardiomyocytes. (A) Relative secreted level of LDH in H9c2 cells treated with different doses of chemerin. (B) The relative level of $\mathrm{LDH}$ in $100 \mathrm{ng} / \mathrm{mL}$ chemerin-treated $\mathrm{H} 9 \mathrm{c} 2$ cells for different lengths of time. Data were presented as means $\pm \mathrm{SEM}$ from three independent experiments. ${ }^{*} p<0.05,{ }^{* *} p<0.01$ vs. Ctrl; ${ }^{\#} p<0.05$ vs. $10 \mathrm{ng} / \mathrm{mL}$ chemerin; ${ }^{\S} p<0.05$ vs. chemerin treatment for $12 \mathrm{~h}$.

TUNEL, and LDH assays, was lower among CMKLR1-siRNAtreated cells than among NCs (Figures 7A-D; all $p<0.01$ ). A similar result was observed in the NLRP3-siRNA-treated group (Figures 7A-D; $p<0.05 \sim p<0.01$ ). Treatment with siRNAs targeting both CMKLR1 and NLRP3 further decreased the ratio of dead cells compared with treatment with either siRNA alone, as determined by EthD-III staining (Figures 7A,B; both $p<0.05$ ).

\section{DISCUSSION}

In this study, we found that chemerin could induce inflammation and cardiomyocyte pyroptosis through its receptor, CMKLR1, and these effects were associated with NLRP3-mediated IL-1 $\beta$ and caspase-1 activation. These results indicated that the chemerin/CMKLR1 axis could promote DCM progression and may represent a new therapeutic target for DCM treatment.

In this study, we successfully generated a rat model of DCM that showed the characteristics of cardiac remodeling, cell death, and cardiac dysfunction. The serum chemerin level has been shown to be an important link between inflammation and diabetes-related complications (Zhang et al., 2018; Shang et al., 2019). Consistent with these observations, we found that chemerin expression was upregulated in the serum and left ventricle myocardium of DCM rats. CMKLR1, a wellcharacterized membrane receptor for chemerin, was also highly expressed in DCM rats and its expression overlapped with that of chemerin. To explore the role of the chemerin/CMKLR1 axis in DCM, we used CMLR1-siRNA in vivo to investigate whether inhibition of CMKLR1 could alleviate DCM progression. Because previous studies have shown that cardiac dysfunction in diabetic rats occurs 8 weeks after STZ injection (Lacombe et al., 2007; Ti et al., 2011), we performed CMKLR1-siRNA treatment in vivo 8 weeks after STZ injection to explore whether inhibition of CMKLR1 exerted a therapeutic effect on DCM. Four weeks after CMKLR1-siRNA injection, analysis of transfection effectiveness showed that the lentiviral CMKLR1-siRNA vector had worked. No notable adverse effects were observed in lentiviral siRNA-treated rats. CMKLR1 and NLRP3 protein expression was significantly suppressed by CMKLR1-siRNA treatment. Moreover, the expression levels of mature caspase1 and IL-1 $\beta$, critical regulators of pyroptosis and inflammation (Lacey et al., 2018; Kelley et al., 2019), were also significantly decreased in CMKLR1-siRNA-treated diabetic rats.

Downregulation of CMKLR1 suppressed cardiac cell death in DCM rats. Although apoptosis and necrosis are the forms of cell death that are normally detected in DCM patients (Chowdhry et al., 2007; D’Arcy, 2019; Ge et al., 2019; Qi et al., 2020) recent studie have shown that other types of cell death, such as pyroptosis, are also found in DCM (Wang X. et al., 2019). Pyroptosis is a type of programmed cell death that is commonly induced by caspase- 1 activation, and is characterized by pore formation on the plasma membrane, cell swelling, nuclear DNA damage, and release of intracellular inflammatory content (Liu et al., 2016; Lacey et al., 2018). Notably, we observed the characteristic hallmarks of pyroptosis in the left ventricles of DCM rats, including cytoplasmic swelling, nuclear DNA damage, and increased activation of caspase-1. This result suggested that pyroptosis had occurred during DCM progression.

In vitro, $\mathrm{DCM}$ is often imitated in rat embryonic heart derived H9C2 cells, neonatal cardiomyocytes or cardiac fibroblasts with high glucose or other kind of cytokine stimulation. H9c2 cells were used in our study to explore the mechanism of chemerin-regulated cell death. Chemerin induced the release of $\mathrm{LDH}$ from $\mathrm{H} 9 \mathrm{c} 2$ cells, indicating that the cell membrane was disrupted. NLRP3 inflammasome activation led to caspase-1 activation, along with pore formation in the plasma membrane and nuclear DNA damage in chemerin-treated H9c2 cells. These data supported our hypothesis that pyroptosis occurred in cardiomyocytes. The TUNEL result indicated that apoptosis also contributed to the DCM-induced cell death. Knockdown 


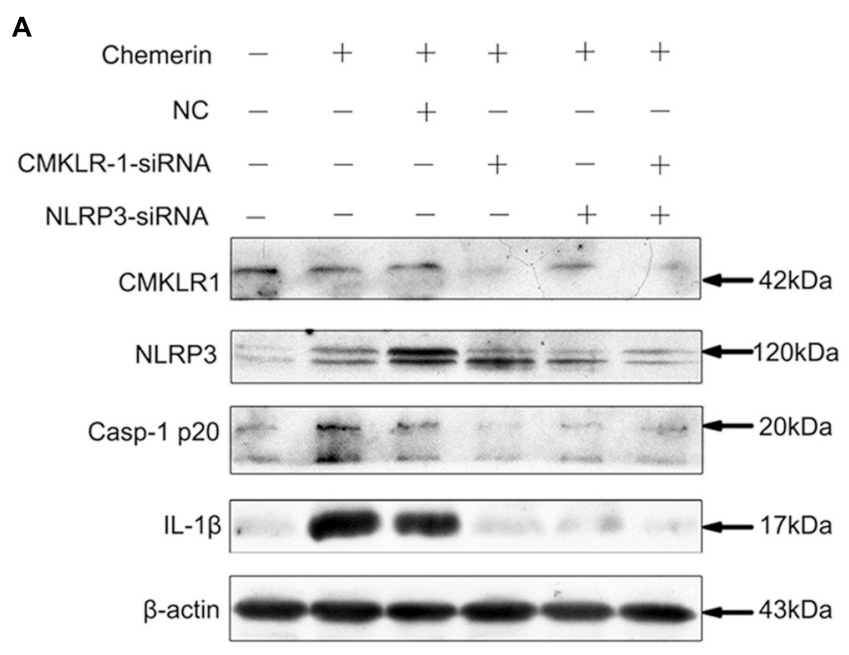

B

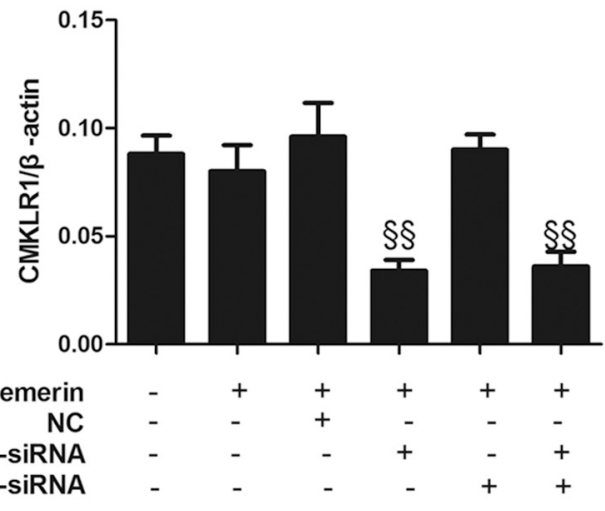

D

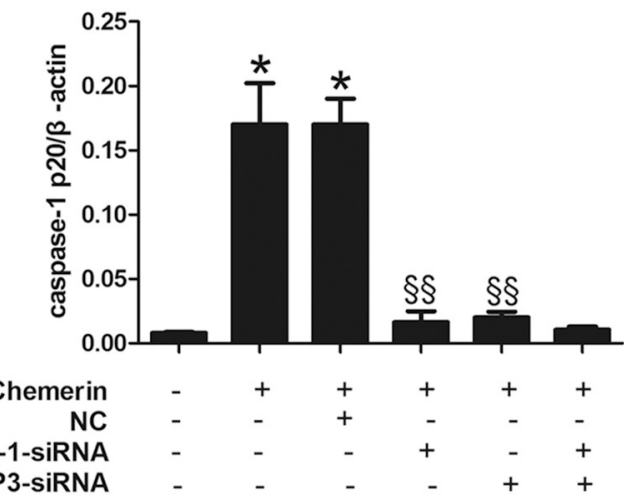

C

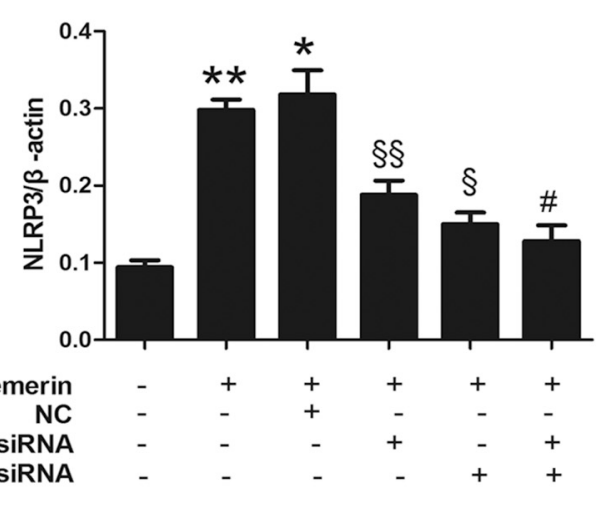

E

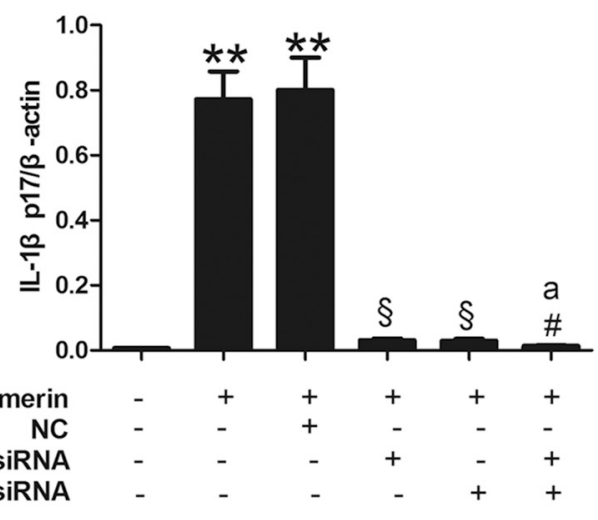

FIGURE 6 | CMKLR1 and NLRP3 induced caspase-1 and IL-1 $\beta$ activation. Relative protein levels of CMKLR1 (A,B), NLRP3 (A,C), activated caspase-1 (A,D), and mature IL-1 $\beta(\mathbf{A}, \mathbf{E})$ in treated $\mathrm{H} 9 \mathrm{c} 2$ cells. Data were presented as means $\pm \mathrm{SEM}$ from three independent experiments. ${ }^{\star} p<0.05,{ }^{\star \star} p<0.01 \mathrm{vs}$. Ctrl; ${ }^{\S} p<0.05,{ }^{\S \varsigma}$ $p<0.01$ vs. Chemerin+NC; ${ }^{\#} p<0.05$ vs. Chemerin+CMKLR1-siRNA; ${ }^{a} p<0.05$ vs. Chemerin+NLRP3-siRNA; NC, negative control.

of CMKLR1 in vivo and in vitro markedly inhibited caspase1 activation, which was accompanied by decreased pyroptosis. These results indicated that caspase-1-dependent pyroptosis is a contributing factor to chemerin-induced cell death in DCM. The chemerin/CMKLR1 axis was shown to be a critical regulator of pyroptosis in DCM. The role of the chemerin/CMKLR1 axis in apoptosis requires further investigation.
The DCM rats showed myocardial lipid accumulation, which can result in cardiac lipotoxicity (Li et al., 2019) and was thus likely to have contributed to DCM. After CMKLR1 downregulation, the cardiac lipid imbalance was markedly reversed.

DCM rats showed substantial hypertrophy and fibrosis in the myocardium as determined by H\&E and Masson's staining. 


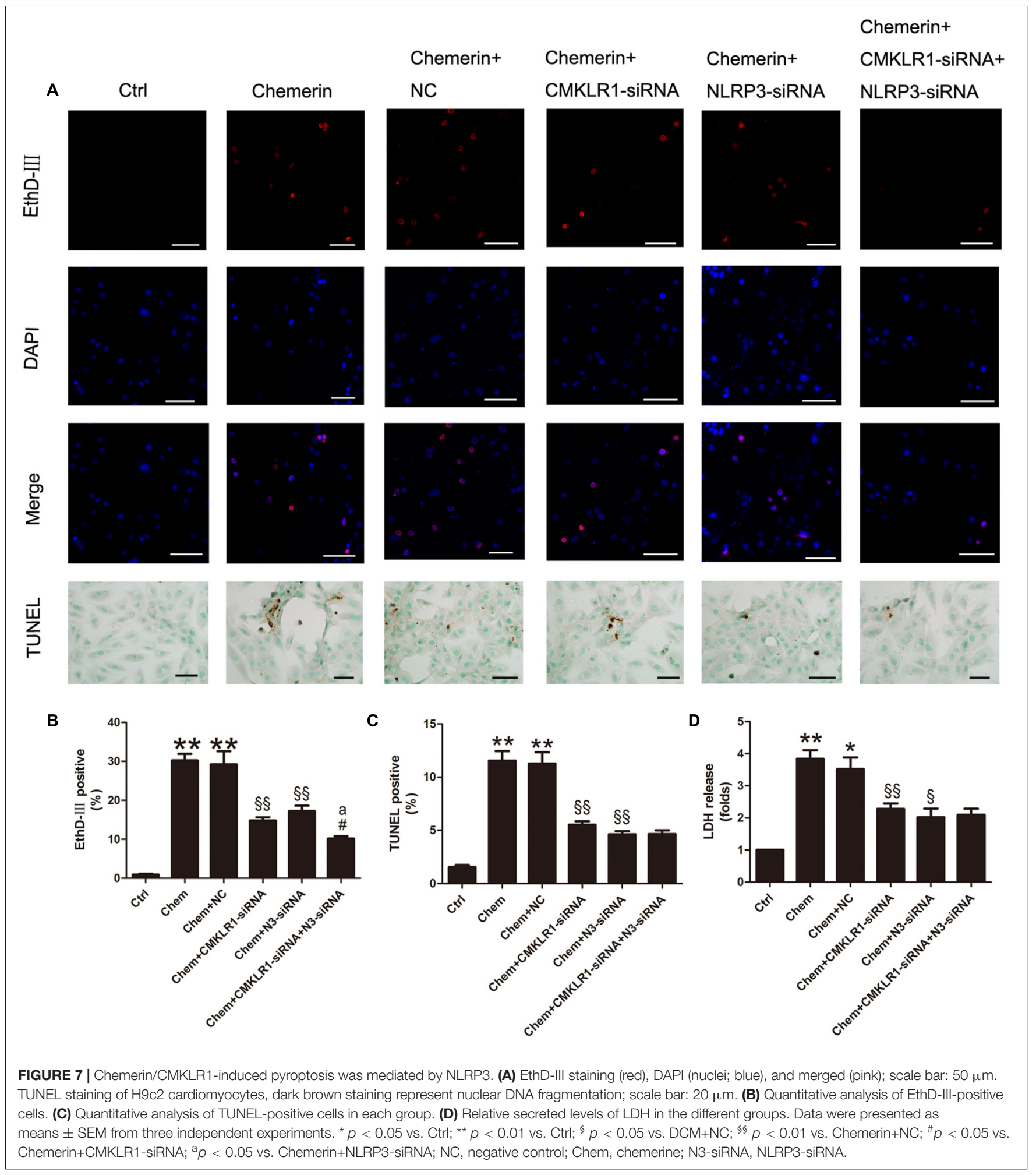

Cardiac hypertrophy and fibrosis, common pathological changes in DCM, can induce left ventricular stiffness (Wang Y. et al., 2019; Yuan et al., 2019; Luo et al., 2020). Consistent with this, diastolic and systolic dysfunction were observed by echocardiographic analysis in DCM rats. Knockdown of CMKLR1 alleviated the aberrant hypertrophy and fibrosis in DCM. Furthermore, both the systolic and diastolic dysfunction of the left ventricle were improved in CMKLR1-siRNA-treated DCM rats, as evidenced by the attenuated hypertrophy, reduced fibrosis, decreased cardiac pyroptosis, and reduced myocardial lipid deposition. These 
observations showed that CMKLR1-siRNA treatment exerts a therapeutic effect on DCM.

The chemerin/CMKLR1 axis has been proposed to regulate the activation of the NLRP3 inflammasome in Kupffer cells in a mouse model of nonalcoholic fatty liver disease (Zhang et al., 2017), and a rat model of limb ischemia/reperfusionacute lung injury (Zou et al., 2019). In line with these findings, our study showed that chemerin promoted caspase1 and IL-1 $\beta$ activation in $\mathrm{H} 9 \mathrm{c} 2$ cardiomyocytes, which could be markedly suppressed by treatment with CMKLR1siRNA. The results indicated that chemerin induced NLRP3 inflammasome activation through the CMKLR1 receptor. Chemerin-induced mature caspase- 1 and IL- $1 \beta$ overexpression was suppressed following NLRP3-siRNA treatment, indicating that NLRP3 is an important mediator of chemerin/CMKLR1regulated inflammasome activation. Interestingly, treatment with siRNAs targeting both CMKLR1 and NLRP3 suppressed the expression of mature IL-1 $\beta$, but not that of caspase1, to a greater extent than treatment with either siRNA alone. These results indicated that CMKLR1 is an important, although not exclusive, mediator of chemerin-induced NLRP3 inflammasome activation, and that the chemerin/CMKLR1 axis may regulate caspase- 1 and IL- $1 \beta$ activation primarily through NLRP3. The EthD-III staining, LDH release assay, and TUNEL staining results indicated that both CMKLR1-siRNA and NLRP3-siRNA treatments could attenuate cell membrane and nuclear DNA damage. Interestingly, EthD-III staining also revealed that the rate of pyroptosis with CMKLR1+NLRP3 knockdown was decreased to a greater extent than with knockdown of either CMKLR1 or NLRP3 alone. These results indicated that NLRP3 inflammasome-regulated caspase-1 was a critical but not exclusive link between the chemerin/CMKLR1 axis and pyroptosis.

In conclusion, our results revealed that the chemerin/CMKLR1 axis played an important role in the inflammation, hypertrophy, pyroptosis, and fibrosis that occurs in the heart tissue of DCM rats. The effect of the chemerin/CMKLR1 axis on inflammation and pyroptosis was mainly mediated by the NLRP3 inflammasome. Caspase-1-regulated pyroptosis played

\section{REFERENCES}

Chowdhry, M. F., Vohra, H. A., and Galinanes, M. (2007). Diabetes increases apoptosis and necrosis in both ischemic and nonischemic human myocardium: role of caspases and poly-adenosine diphosphate-ribose polymerase. J. Thorac. Cardiovasc. Surg. 134, 124-131.e3. doi: 10.1016/j.jtcvs.2006.12.059

D'Arcy, M. S. (2019). Cell death: a review of the major forms of apoptosis, necrosis and autophagy. Cell Biol. Int. 43, 582-592. doi: 10.1002/cbin.11137

Deckx, S., Heggermont, W., Carai, P., Rienks, M., Dresselaers, T., Himmelreich, U., et al. (2018). Osteoglycin prevents the development of age-related diastolic dysfunction during pressure overload by reducing cardiac fibrosis and inflammation. Matrix Biol. 66, 110-124. doi: 10.1016/j.matbio.2017.09.002

Ding, S., Liu, D., Wang, L., Wang, G., and Zhu, Y. (2019). Inhibiting microRNA29a protects myocardial ischemia-reperfusion injury by targeting SIRT1 and regulating NLRP3 and apoptosis pathway. J. Pharmacol. Exp. Ther. 372, 128135. doi: 10.1124/jpet.119.256982

Ge, Z. D., Lian, Q., Mao, X., and Xia, Z. (2019). Current status and challenges of NRF2 as a potential therapeutic target for diabetic cardiomyopathy. Int. Heart J. 60, 512-520. doi: 10.1536/ihj.18-476 an important role in the pathogenesis of DCM. Gene interference targeting CMKLR1 seems to be effective for treating DCM in a rat model. Further studies are needed to validate the targeting of chemerin/CMKLR1 as a potential therapeutic strategy for the treatment of DCM.

\section{DATA AVAILABILITY STATEMENT}

The raw data supporting the conclusions of this manuscript will be made available by the authors, without undue reservation, to any qualified researcher.

\section{ETHICS STATEMENT}

The study protocol was approved by the Institutional Animal Care and Use Committee of the Guangxi Medical University.

\section{AUTHOR CONTRIBUTIONS}

$\mathrm{BL}$ and YX were responsible to induce animal model and cell experiment. YH performed immunohistochemistry staining and other staining. XL analyzed and interpreted the animal model data. HQ performed the ultrasonic cardiogram examination of the heart. MW carried out western blot experiment. BL conceived the manuscript. All authors read and approved the final manuscript.

\section{FUNDING}

This work was supported by the National Natural Science Foundation of China (grant no. 81600185), the Province Natural Science Foundation of Guangxi (grant no. 2018JJB140299), and the "Medical Excellence Award" Funded by the Creative Research Development Grant from the First Affiliated Hospital of Guangxi Medical University.

Gu, J., Huang, W., Zhang, W., Zhao, T., Gao, C., Gan, W., et al. (2019). Sodium butyrate alleviates high-glucose-induced renal glomerular endothelial cells damage via inhibiting pyroptosis. Int. Immunopharmacol. 75:105832. doi: 10. 1016/j.intimp.2019.105832

Helfer, G., and Wu, Q. F. (2018). Chemerin: a multifaceted adipokine involved in metabolic disorders. J. Endocrinol. 238, R79-R94. doi: 10.1530/JOE-180174

Jin, X., Chen, C., Li, D., Su, Q., Hang, Y., Zhang, P., et al. (2017). PRDX2 in myocyte hypertrophy and survival is mediated by TLR4 in acute infarcted myocardium. Sci. Rep. 7:6970. doi: 10.1038/s41598-017-06 718-7

Kadoglou, N. P., Tahmatzidis, D. K., Giannakoulas, C., Kapelouzou, A., Gkontopoulos, A., Parissis, J., et al. (2015). Serum levels of novel adipokines, omentin-1 and chemerin, in patients with acute myocardial infarction: KOZANI STUDY. J. Cardiovasc. Med. (Hagerstown) 16, 341-346. doi: 10.2459/JCM.0000000000000053

Kaur, J., Mattu, H. S., Chatha, K., and Randeva, H. S. (2018). Chemerin in human cardiovascular disease. Vascul. Pharmacol. 110, 1-6. doi: 10.1016/j.vph.2018. 06.018 
Kelley, N., Jeltema, D., Duan, Y., and He, Y. (2019). The NLRP3 inflammasome: an overview of mechanisms of activation and regulation. Int. J. Mol. Sci. 20:3328. doi: 10.3390/ijms20133328

Lacey, C. A., Mitchell, W. J., Dadelahi, A. S., and Skyberg, J. A. (2018). Caspase-1 and Caspase-11 mediate pyroptosis, inflammation, and control of brucella joint infection. Infect. Immun. 86:e00361-18. doi: 10.1128/IAI.00361-18

Lacombe, V. A., Viatchenko-Karpinski, S., Terentyev, D., Sridhar, A., Emani, S., Bonagura, J. D., et al. (2007). Mechanisms of impaired calcium handling underlying subclinical diastolic dysfunction in diabetes. Am. J. Physiol. Regul. Integr. Comp. Physiol. 293, R1787-R1797. doi: 10.1152/ajpregu.00059.2007

Li, H., Fan, J., Zhao, Y., Zhang, X., Dai, B., Zhan, J., et al. (2019). Nuclear miR320 mediates diabetes-induced cardiac dysfunction by activating transcription of fatty acid metabolic genes to cause lipotoxicity in the heart. Circ. Res. 125, 1106-1120. doi: 10.1161/CIRCRESAHA.119.314898

Liu, X., Zhang, Z., Ruan, J., Pan, Y., Magupalli, V. G., Wu, H., et al. (2016). Inflammasome-activated gasdermin $\mathrm{D}$ causes pyroptosis by forming membrane pores. Nature 535, 153-158. doi: 10.1038/nature18629

Luo, J., Yan, D., Li, S., Liu, S., Zeng, F., Cheung, C. W., et al. (2020). Allopurinol reduces oxidative stress and activates Nrf2/p62 to attenuate diabetic cardiomyopathy in rats. J. Cell Mol. Med. 24, 1760-1773. doi: 10.1111/jcmm. 14870

Mariani, F., and Roncucci, L. (2015). Chemerin/chemR23 axis in inflammation onset and resolution. Inflamm. Res. 64, 85-95. doi: 10.1007/s00011-014-0792-7

Marwick, T. H., Ritchie, R., Shaw, J. E., and Kaye, D. (2018). Implications of underlying mechanisms for the recognition and management of diabetic cardiomyopathy. J. Am. Coll. Cardiol. 71, 339-351. doi: 10.1016/j.jacc.2017. 11.019

Meng, L., Lin, H., Zhang, J., Lin, N., Sun, Z., Gao, F., et al. (2019). Doxorubicin induces cardiomyocyte pyroptosis via the TINCR-mediated posttranscriptional stabilization of NLR family pyrin domain containing 3. J. Mol. Cell Cardiol. 136, 15-26. doi: 10.1016/j.yjmcc.2019.08.009

Neves, K. B., Montezano, A. C., Alves-Lopes, R., Bruder-Nascimento, T., Costa, R. M., Costa, R. S., et al. (2018a). Upregulation of Nrf2 and decreased redox signaling contribute to renoprotective effects of chemerin receptor blockade in diabetic mice. Int. J. Mol. Sci. 19:2454. doi: 10.3390/ijms19082454

Neves, K. B., Nguyen Dinh Cat, A., Alves-Lopes, R., Harvey, K. Y., Costa, R. M. D., Lobato, N. S., et al. (2018b). Chemerin receptor blockade improves vascular function in diabetic obese mice via redox-sensitive and Akt-dependent pathways. Am. J. Physiol. Heart Circ. Physiol. 315, H1851-H1860. doi: 10.1152/ ajpheart.00285.2018

Ogurtsova, K., da Rocha Fernandes, J. D., Huang, Y., Linnenkamp, U., Guariguata, L., Cho, N. H., et al. (2017). IDF diabetes atlas: global estimates for the prevalence of diabetes for 2015 and 2040. Diabetes Res. Clin. Pract. 128, 40-50. doi: 10.1016/j.diabres.2017.03.024

Pan, J., Han, L., Guo, J., Wang, X., Liu, D., Tian, J., et al. (2018). AIM2 accelerates the atherosclerotic plaque progressions in ApoE-/- mice. Biochem. Biophys. Res. Commun. 498, 487-494. doi: 10.1016/j.bbrc.2018.03.005

Qi, B., He, L., Zhao, Y., Zhang, L., He, Y., Li, J., et al. (2020). Akap1 deficiency exacerbates diabetic cardiomyopathy in mice by NDUFS1-mediated mitochondrial dysfunction and apoptosis. Diabetologia 63, 1072-1087. doi: 10. 1007/s00125-020-05103-w

Rodriguez-Penas, D., Feijoo-Bandin, S., Garcia-Rua, V., Mosquera-Leal, A., Duran, D., Varela, A., et al. (2015). The adipokine chemerin induces apoptosis in cardiomyocytes. Cell Physiol. Biochem. 37, 176-192. doi: 10.1159/000430343

Shang, J., Wang, L., Zhang, Y., Zhang, S., Ning, L., Zhao, J., et al. (2019). Chemerin/ChemR23 axis promotes inflammation of glomerular endothelial cells in diabetic nephropathy. J. Cell Mol. Med. 23, 3417-3428. doi: 10.1111/ jcmm.14237

Stojek, M. (2017). The role of chemerin in human disease. Postepy. Hig. Med. Dosw. (Online) 71, 110-117. doi: 10.5604/01.3001.0010.3795
Ti, Y., Xie, G. L., Wang, Z. H., Bi, X. L., Ding, W. Y., Wang, J., et al. (2011). TRB3 gene silencing alleviates diabetic cardiomyopathy in a type 2 diabetic rat model. Diabetes Metab. Res. Rev. 60, 2963-2974. doi: 10.2337/db11-0549

Wang, X., Pan, J., Liu, H., Zhang, M., Liu, D., Lu, L., et al. (2019). AIM2 gene silencing attenuates diabetic cardiomyopathy in type 2 diabetic rat model. Life Sci. 221, 249-258. doi: 10.1016/j.lfs.2019.02.035

Wang, Y., Li, H., Li, Y., Zhao, Y., Xiong, F., Liu, Y., et al. (2019). Coriolus versicolor alleviates diabetic cardiomyopathy by inhibiting cardiac fibrosis and NLRP3 inflammasome activation. Phytother. Res. 33, 2737-2748. doi: 10.1002/ptr.6448

Wang, Z., Yu, J., Wu, J., Qi, F., Wang, H., Wang, Z., et al. (2016). Scutellarin protects cardiomyocyte ischemia-reperfusion injury by reducing apoptosis and oxidative stress. Life Sci. 157, 200-207. doi: 10.1016/j.lfs.2016.01.018

Wu, B., Huang, X. Y., Li, L., Fan, X. H., Li, P. C., Huang, C. Q., et al. (2019). Attenuation of diabetic cardiomyopathy by relying on kirenol to suppress inflammation in a diabetic rat model. J. Cell Mol. Med. 23, 7651-7663. doi: $10.1111 /$ jcmm.14638

Yang, F., Qin, Y., Wang, Y., Meng, S., Xian, H., Che, H., et al. (2019). Metformin inhibits the NLRP3 inflammasome via AMPK/mTOR-dependent effects in diabetic cardiomyopathy. Int. J. Biol. Sci. 15, 1010-1019. doi: 10.7150/ijbs.29680

Yang, L., Wang, S., Ma, J., Li, J., Yang, J., Bucala, R., et al. (2019). CD74 knockout attenuates alcohol intake-induced cardiac dysfunction through AMPK-Skp2mediated regulation of autophagy. Biochim. Biophys. Acta Mol. Basis Dis. 1865, 2368-2378. doi: 10.1016/j.bbadis.2019.05.020

Yap, J., Tay, W. T., Teng, T. K., Anand, I., Richards, A. M., Ling, L. H., et al. (2019). Association of diabetes mellitus on cardiac remodeling, quality of life, and clinical outcomes in heart failure with reduced and preserved ejection fraction. J. Am. Heart Assoc. 8:e013114. doi: 10.1161/JAHA.119.013114

Yuan, H., Fan, Y., Wang, Y., Gao, T., Shao, Y., Zhao, B., et al. (2019). Calciumsensing receptor promotes high glucoseinduced myocardial fibrosis via upregulation of the TGFbeta1/Smads pathway in cardiac fibroblasts. Mol. Med. Rep. 20, 1093-1102. doi: 10.3892/mmr.2019.10330

Zhang, W., Liu, Y., Wu, M., Zhu, X., Wang, T., He, K., et al. (2017). PI3K inhibition protects mice from NAFLD by down-regulating CMKLR1 and NLRP3 in Kupffer cells. J. Physiol. Biochem. 73, 583-594. doi: 10.1007/s13105-0170589-6

Zhang, X., Fu, Y., Li, H., Shen, L., Chang, Q., Pan, L., et al. (2018). H3 relaxin inhibits the collagen synthesis via ROS- and P2X7R-mediated NLRP3 inflammasome activation in cardiac fibroblasts under high glucose. J. Cell Mol. Med. 22, 1816-1825. doi: 10.1111/jcmm.13464

Zhang, Z., Wang, J., and Wang, H. (2018). Correlation of blood glucose, serum chemerin and insulin resistance with NAFLD in patients with type 2 diabetes mellitus. Exp. Ther. Med. 15, 2936-2940. doi: 10.3892/etm.2018.5753

Zhou, X., Tao, Y., Chen, Y., Xu, W., Qian, Z., and Lu, X. (2019). Serum chemerin as a Novel prognostic indicator in chronic heart failure. J. Am. Heart Assoc. 8:e012091. doi: 10.1161/JAHA.119.012091

Zou, R., Wang, M. H., Chen, Y., Fan, X., Yang, B., Du, J., et al. (2019). Hydrogenrich saline attenuates acute lung injury induced by limb ischemia/reperfusion via down-regulating chemerin and NLRP3 in rats. Shock 52, 134-141. doi: 10.1097/SHK.0000000000001194

Conflict of Interest: The authors declare that the research was conducted in the absence of any commercial or financial relationships that could be construed as a potential conflict of interest.

Copyright (c) 2020 Xie, Huang, Ling, Qin, Wang and Luo. This is an open-access article distributed under the terms of the Creative Commons Attribution License (CC BY). The use, distribution or reproduction in other forums is permitted, provided the original author(s) and the copyright owner(s) are credited and that the original publication in this journal is cited, in accordance with accepted academic practice. No use, distribution or reproduction is permitted which does not comply with these terms. 